BN-TH-98-14

NTZ 17/98

hep-th/9807088

\title{
Gauge parameter dependence in the background field gauge and the construction of an invariant charge
}

\author{
Rainer Häußling*, Elisabeth Kraus ${ }^{\dagger}$, Klaus Sibold* \\ *Institut für Theoretische Physik und \\ Naturwissenschaftlich-Theoretisches Zentrum, Universität Leipzig \\ Augustusplatz 10/11, D-04109 Leipzig, Germany \\ ${ }^{\dagger}$ Physikalisches Institut, Universität Bonn, Nußallee 12, D-53115 Bonn, Germany
}

\begin{abstract}
By using the enlarged BRS transformations we control the gauge parameter dependence of Green functions in the background field gauge. We show that it is unavoidable - also if we consider the local Ward identity - to introduce the normalization gauge parameter $\xi_{o}$, which enters the Green functions of higher orders similarly to the normalization point $\kappa$. The dependence of Green functions on $\xi_{o}$ is governed by a further partial differential equation. By modifying the Ward identity we are able to construct in 1-loop order a gauge parameter independent combination of 2-point vector and background vector functions. By explicit construction of the next orders we show that this combination can be used to construct a gauge parameter independent RG-invariant charge. However, it is seen that this RG-invariant charge does not satisfy the differential equation of the normalization gauge parameter $\xi_{o}$, and, hence, is not $\xi_{o}$-independent as required.
\end{abstract}

PACS number(s): 11.10.Gh, 11.15.-q

Keywords: $\quad$ Quantum Field Theory, Background Field Gauge, Local Ward Identity, Invariant Charge 


\section{Introduction}

Gauge invariance is an issue of central importance in gauge theories: physical quantities have to be gauge invariant. In perturbative calculations gauge invariance is to be accompanied by gauge parameter independence. A quantity qualifies as observable only if it is gauge invariant and independent of gauge parameters used for the construction of Green functions. In concrete calculations one often uses gauge parameter independence as a hint that an object under study might indeed be physical (although gauge parameter independence clearly is only a necessary condition). In practice the discussion of (in)dependence is inevitably linked with questions of scheme dependence - meaning two different things: the way one is removing divergencies and the normalization conditions one chooses for fixing the free parameters.]

If one formulates theories purely with the help of Ward identities, Slavnov-Taylor identities and the like - as we shall do below - one proceeds independently from any scheme and thus has disentangled these questions. Making in addition vary the gauge parameters (into Grassmann variables) [1] and taking that as a contribution to the Slavnov-Taylor identity one can also control gauge parameter dependence algebraically, i.e. independently from any scheme [2].

We shall perform such a study when also background gauge fields are present and - in order not to be academic - treat a well-defined problem: the construction of an invariant charge in Yang-Mills theory. The use of background fields [3, 4 has proven to be fruitful: once the Slavnov-Taylor identity has been established (or is considered to hold in a naive form) one can simplify the calculation of renormalization constants because one is able to dispose over a fairly naive local Ward identity (see e.g. [5]). Equalities among these constants suggest e.g. to translate properties of the effective charge from QED to YangMills [6]. Findings in this direction have, of course, to be compared with results of the pinch technique [0, 8] which yields gauge parameter independent self-energies, thus candidates for observables and potentially having the interpretation of an invariant charge [6]. Since one is, however, manipulating diagrams explicitly it is not obvious how these notions can be extended to higher orders. Similarly in the work of Watson [6] a specific (ghost free) gauge is chosen, such that there the "analytic-algebraic" scheme independent characterization of an effective charge is missing, too.

Before describing our procedure we would like to point out that the existence of an effective charge is very important indeed. In renormalon calculations it is tacitly assumed [9]; it enters in all questions of improvement and scheme dependence of perturbative

\footnotetext{
${ }^{1}$ These two questions can interfere with each other. Minimal subtractions, for instance, do both: remove divergencies and set implicitly normalization conditions.
} 
contributions of finite order, and, in fact, becomes there an issue of experimental relevance: if one "improves" the explicit calculation of some finite order by replacing the coupling by the running coupling and compares with experiment one faces the question of gauge parameter dependence. And thus a gauge independent effective charge would obviously be of practical relevance.

The present paper is structured as follows: First we recall the notion of the effective charge in QED by stressing those points which are needed in an extension to the nonabelian case (section 2). Next we look at the local Ward identity of Yang-Mills theory and introduce external fields (background fields) in such a way that the correspondingly changed Ward identity can be proved to all orders (sections 3, 4, 5). By varying the gauge parameter and taking this variation into account in the Slavnov-Taylor identity we provide the basis for the construction of an effective charge made out of 2-point functions. We show that such an object can be constructed (scheme independently) as being gauge independent and also invariant under the renormalization group (sections 6,7). But it turns out that it does not satisfy an independence equation with respect to a normalization gauge parameter $\xi_{0}$ (section 8) which has to be introduced in the course of defining the theory. We end with some conclusions (section 9).

\section{Properties and construction of an invariant charge}

In renormalized perturbation theory the QED Green functions to all orders are uniquely determined by the gauge Ward identity

$$
\left(e \mathbf{w}_{e m}-\partial^{\nu} \frac{\delta}{\delta A^{\nu}}\right) \Gamma=-\frac{1}{\xi} \square \partial A
$$

with

$$
\mathbf{w}_{e m}=-i \sum_{f} Q_{f}\left(\bar{f} \frac{\vec{\delta}}{\delta \bar{f}}-\frac{\overleftarrow{\delta}}{\delta f} f\right)
$$

and normalization conditions to be imposed on the photon and fermion self-energy in order to fix masses and residua of the propagators. Explicitly we impose:

$$
\begin{array}{rlrl}
\Gamma_{A A}^{T}\left(p^{2}=0\right) & =0 & & \left.\frac{1}{p^{2}} \Gamma_{A A}^{T}\left(p^{2}\right)\right|_{p^{2}=\kappa^{2}}=1 \\
\left.\Gamma_{\bar{f} f}\right|_{\not p=m_{f}}=0 & & \left.\partial_{p} \Gamma_{\bar{f} f}\right|_{\not p=\kappa}=1
\end{array}
$$

$\Gamma$ denotes the generating functional of 1 particle irreducible (1PI) Green functions. From $\Gamma$ the vertex functions are determined by differentiation with respect to the classical fields, 
for example:

$$
\Gamma_{A^{\mu} A^{\nu}}(x, y) \equiv \frac{\delta^{2} \Gamma}{\delta A^{\mu}(x) \delta A^{\nu}(y)}
$$

In (2.3) we have decomposed the vector boson 2-point function into the transversal part $\Gamma_{A A}^{T}\left(p^{2}\right)$ and the longitudinal part $\Gamma_{A A}^{L}\left(p^{2}\right)$ :

$$
\Gamma_{A^{\mu} A^{\nu}}=\left(\eta^{\mu \nu}-\frac{p^{\mu} p^{\nu}}{p^{2}}\right) \Gamma_{A A}^{T}\left(p^{2}\right)+\frac{p^{\mu} p^{\nu}}{p^{2}} \Gamma_{A A}^{L}\left(p^{2}\right)
$$

In the perturbative expansion the lowest order of the generating functional of 1PI Green functions is the classical action:

$$
\begin{aligned}
& \Gamma_{c l}=\int \mathrm{d}^{4} x\left\{-\frac{1}{4} F^{\mu \nu} F_{\mu \nu}-\frac{1}{2 \xi}(\partial A)^{2}\right. \\
&\left.+\sum_{f}\left(i \bar{f} \gamma^{\mu} \partial_{\mu} f-m_{f} \bar{f} f+e Q_{f} \bar{f} \gamma^{\mu} A_{\mu} f\right)\right\}
\end{aligned}
$$

For all further considerations it is important to note that the normalization of the coupling constant $e$ is determined by the Ward identity. As a consequence, in invariant schemes the wave-function normalization $z_{A}$ of the photon field is related to the normalization $z_{e}$ of the coupling constant according to the well-known relation

$$
z_{e}^{2} z_{A}=1
$$

In the general approach, which does not rely on properties of invariant schemes, but just on properties of finite renormalized Green functions, the scheme dependent relation (2.9) is reflected in relations between the coefficient functions of the Callan-Symanzik (CS) equation,

$$
\left(\kappa \partial_{\kappa}+m_{f} \partial_{m_{f}}+\beta_{e} e \partial_{e}-\gamma_{A}\left(\int \mathrm{d}^{4} x\left(A \frac{\delta}{\delta A}\right)+2 \xi \partial_{\xi}\right)-\gamma_{f} \int \mathrm{d}^{4} x\left(\bar{f} \frac{\vec{\delta}}{\delta \bar{f}}+\frac{\overleftarrow{\delta}}{\delta f} f\right)\right) \Gamma=\left[\Delta_{m}\right]_{3}^{3} \cdot \Gamma
$$

and the renormalization group $(\mathrm{RG})$ equation,

$$
\left(\kappa \partial_{\kappa}+\tilde{\beta}_{e} e \partial_{e}-\tilde{\gamma}_{A}\left(\int \mathrm{d}^{4} x\left(A \frac{\delta}{\delta A}\right)+2 \xi \partial_{\xi}\right)-\tilde{\gamma}_{f} \int \mathrm{d}^{4} x\left(\bar{f} \frac{\vec{\delta}}{\delta \bar{f}}+\frac{\overleftarrow{\delta}}{\delta f} f\right)\right) \Gamma=0
$$

with

$$
\tilde{\beta}_{e}=\beta_{e}, \quad \tilde{\gamma}_{A}=\gamma_{A} \quad \text { if } \quad \kappa^{2} \rightarrow-\infty .
$$

Indeed, by applying these equations to the Ward identity (2.1) one obtains that the anomalous dimension of the photon is equal to the $\beta$-function of the respective equation:

$$
\beta_{e}=\gamma_{A} \quad \tilde{\beta}_{e}=\tilde{\gamma}_{A}
$$


Finally, gauge parameter independence of the $\beta$-functions $\beta_{e}$ and $\tilde{\beta}_{e}$ and also of the transversal photon 2-point function is derived from the Ward identity and the normalization condition imposed on the photon self-energy (2.3). Therefore the inverse transversal photon self-energy is an appropriate object for defining the invariant charge of QED:

$$
Q_{e}\left(p^{2}, m_{f}^{2}, \kappa^{2}\right)=\frac{p^{2} e^{2}}{\Gamma_{A A}^{T}\left(p^{2}\right)}=\frac{e^{2}}{1+\Pi\left(p^{2}\right)} \quad \text { and } \quad \Gamma_{A A}^{T}\left(p^{2}\right) \equiv p^{2}\left(1+\Pi\left(p^{2}\right)\right)
$$

$Q_{e}\left(p^{2}, m_{f}^{2}, \kappa^{2}\right)$ is gauge parameter independent,

$$
\partial_{\xi} Q_{e}\left(p^{2}, m_{f}^{2}, \kappa^{2}\right)=0
$$

well normalized due to $(2.3)$,

$$
\left.Q_{e}\left(p^{2}, m_{f}^{2}, \kappa^{2}\right)\right|_{p^{2}=\kappa^{2}}=e^{2}
$$

and satisfies the homogeneous RG equation:

$$
\left(\kappa \partial_{\kappa}+\beta_{e} e \partial_{e}\right) Q_{e}\left(p^{2}, m_{f}^{2}, \kappa^{2}\right)=0
$$

Applying the CS operator to the invariant charge, it is seen to satisfy also the respective CS equation but with a soft breaking on the right hand side:

$$
\left(\kappa \partial_{\kappa}+m_{f} \partial_{m_{f}}+\beta_{e} e \partial_{e}\right) Q_{e}\left(p^{2}, m_{f}^{2}, \kappa^{2}\right)=\left[\Delta_{m}\right]_{3}^{3} \cdot Q_{e} \stackrel{p^{2} \rightarrow-\infty}{\longrightarrow} 0
$$

with

$$
\left[\Delta_{m}\right]_{3}^{3} \cdot Q_{e} \equiv Q_{e} \frac{\left[\Delta_{m}\right]_{3}^{3} \cdot \Gamma_{A A}^{T}}{\Gamma_{A A}^{T}}=e^{2} p^{2}\left[\Delta_{m}\right]_{3}^{3} \cdot G_{A A}^{T}
$$

$G_{A A}^{T}$ is the transversal part of the full photon propagator.

The RG equation (2.11) can be solved by solving first the homogeneous equation (2.17) and the characteristic equation of the coupling. The solution of the characteristic equations, usually called the running coupling, can be identified with the object $Q_{e}\left(p^{2}, m_{f}^{2}, \kappa^{2}\right)(2.14)$ defined by the photon propagator, and for this reason $Q_{e}$ serves as an invariant charge of QED.

The situation is drastically changed in nonabelian gauge theories: Due to the fact that nonabelian gauge invariance is broken non-linearly in the course of quantization, the relation between the $\beta$-functions and the anomalous dimensions of the vector bosons is lost. But even worse, it is not obvious how to define an invariant charge in terms of off-shell QCD Green functions which can be identified with the running coupling of QCD. Such a construction is even not possible if one wants to define the invariant charge by a combination of interaction vertices and 2 -point functions as it is done in the $\phi^{4}$-theory. 
The point is, that in principle it is not possible to remove the local gauge parameter contributions to the 2- and 3-point functions in 1-loop order at the same time by means of a normalization condition. If one would do so, physical objects like $\beta$-functions and Green functions of invariant operators would not be gauge parameter independent in higher orders of perturbation theory. The technical machinery for controlling the gauge parameter in a scheme-independent way has been provided by the BRS-varying gauge parameter as it was introduced in [2]. For fixing notations and for convenience we shortly want to summarize the results for QCD as presented in this paper, since they are the basic ingredients for understanding the problems concerning the construction of an invariant charge in nonabelian gauge theories.

For the purpose of the paper we restrict ourselves to $\mathrm{SU}(\mathrm{N})$ gauge theories with massless gauge bosons and parity conservation in the fermion sector, as it is the case, for example, in QCD. Invariance under nonabelian gauge transformations

$$
\begin{aligned}
\delta_{\omega} A_{a \mu} & =\partial_{\mu} \omega_{a}+g f_{a b c} A_{b \mu} \omega_{c} \\
\delta_{\omega} \Psi & =-g \omega_{a} T_{a} \Psi
\end{aligned}
$$

determines the Yang-Mills part and the matter part of the classical action:

$$
\begin{aligned}
\Gamma_{Y M} & =-\frac{1}{4} \int \mathrm{d}^{4} x G_{a}^{\mu \nu} G_{a \mu \nu} \\
\Gamma_{\text {matter }} & =\int \mathrm{d}^{4} x\left\{i \bar{\Psi} \gamma^{\mu} D_{\mu} \Psi-M \bar{\Psi} \Psi\right\}
\end{aligned}
$$

with

$$
\begin{aligned}
G_{a}^{\mu \nu} & =\partial^{\mu} A_{a}^{\nu}-\partial^{\nu} A_{a}^{\mu}+g f_{a b c} A_{b}^{\mu} A_{c}^{\nu} \\
D_{\mu} \Psi & =\left(\partial_{\mu}+g A_{a \mu} T_{a}\right) \Psi
\end{aligned}
$$

$T_{a}$ denote the anti-hermitean generators of the fundamental representation, $f_{a b c}$ the structure constants of $\mathrm{SU}(\mathrm{N})$,

$$
\left[T_{a}, T_{b}\right]=f_{a b c} T_{c}
$$

and $\Psi$ are N-component massive Dirac spinors.

When quantizing the theory local gauge invariance (2.20) has to be broken due to the necessity of adding a gauge fixing part $\Gamma_{g . f}$. to the classical action:

$$
\Gamma_{g . f .}=\int \mathrm{d}^{4} x\left\{\frac{\xi}{2} B_{a} B_{a}+B_{a} \partial^{\mu} A_{a \mu}\right\}
$$

Here we have introduced the $(B, \xi)$-gauges, where BRS transformations are nilpotent on all the fields. The fields $B_{a}$ are auxiliary (Lagrange multiplier) fields with dimension 2 
transforming according to the adjoint representation of $\mathrm{SU}(\mathrm{N})$. Eliminating them via their equations of motion leads back to the usual $\xi$-gauges. Nonabelian gauge invariance has to be replaced by BRS symmetry introducing the Faddeev-Popov ghosts $c_{a}, \bar{c}_{a}$ :

$$
\begin{aligned}
\mathrm{s} A_{a \mu}=\partial_{\mu} c_{a}+g f_{a b c} A_{b \mu} c_{c} & , \mathrm{~s} c_{a}=-\frac{1}{2} g f_{a b c} c_{b} c_{c} \\
\mathrm{~s} \bar{c}_{a}=B_{a} & , \mathrm{~s} B_{a}=0 \\
\mathrm{~s} \Psi=-g c_{a} T_{a} \Psi & , \mathrm{s} \bar{\Psi}=g c_{a} \bar{\Psi} T_{a}
\end{aligned}
$$

Following [2] we also transform the gauge parameter $\xi$ into a Grassmann variable $\chi$ :

$$
\mathrm{s} \xi=\chi \quad, \quad \mathrm{s} \chi=0
$$

Enlarging the classical action by the Faddeev-Popov part, which depends on the ghosts and the parameter $\chi$,

$$
\Gamma_{\phi \pi}=\int \mathrm{d}^{4} x\left\{-\bar{c}_{a} \square c_{a}-g f_{a b c} \bar{c}_{a} \partial^{\mu}\left(A_{b \mu} c_{c}\right)+\frac{1}{2} \chi \bar{c}_{a} B_{a}\right\},
$$

the classical action is invariant under the BRS transformations (2.25) and (2.26):

$$
\mathrm{s} \Gamma_{c l}=0 \quad, \quad \Gamma_{c l}=\Gamma_{Y M}+\Gamma_{\text {matter }}+\Gamma_{g . f .}+\Gamma_{\phi \pi}
$$

For this reason the Green functions in higher orders have to be defined by the SlavnovTaylor (ST) identity, instead of using a gauge Ward identity, this ST identity being the functional version of classical BRS symmetry:

$$
\mathcal{S}(\Gamma)=0
$$

with

$$
\mathcal{S}(\Gamma) \equiv \int \mathrm{d}^{4} x\left\{\frac{\delta \Gamma}{\delta \rho_{a}^{\mu}} \frac{\delta \Gamma}{\delta A_{a \mu}}+\frac{\delta \Gamma}{\delta \sigma_{a}} \frac{\delta \Gamma}{\delta c_{a}}+B_{a} \frac{\delta \Gamma}{\delta \bar{c}_{a}}+\Gamma \frac{\overleftarrow{\delta}}{\delta \Psi} \frac{\vec{\delta}}{\delta \bar{Y}} \Gamma+\Gamma \frac{\overleftarrow{\delta}}{\delta Y} \frac{\vec{\delta}}{\delta \bar{\Psi}} \Gamma\right\}+\chi \partial_{\xi} \Gamma
$$

$\Gamma$ is the generating functional of $1 \mathrm{PI}$ Green functions of the $\mathrm{SU}(\mathrm{N})$ gauge theory. The external fields $\rho_{a}^{\mu}, \sigma_{a}, Y$ and $\bar{Y}$ are coupled to the non-linear BRS transformations in (2.25) by adding the external field part $\Gamma_{\text {ext.f. }}$ to the classical action:

$$
\Gamma_{\text {ext.f. }}=\int \mathrm{d}^{4} x\left\{\rho_{a}^{\mu} \mathrm{s} A_{a \mu}+\sigma_{a} \mathrm{~s} c_{a}+\mathrm{s} \bar{\Psi} Y+\bar{Y} \mathrm{~s} \Psi\right\}
$$

The classical action is not only BRS-invariant but also invariant under rigid SU(N) transformations. In symmetric theories it is possible to require rigid $\mathrm{SU}(\mathrm{N})$-invariance by means of a Ward identity to all orders:

$$
\mathcal{W}_{a} \Gamma=0
$$


with

$$
\mathcal{W}_{a} \Gamma \equiv-\int \mathrm{d}^{4} x\left\{\sum_{\Phi} f_{a b c} \Phi_{b} \frac{\delta \Gamma}{\delta \Phi_{c}}+\Gamma \frac{\overleftarrow{\delta}}{\delta \Psi} T_{a} \Psi-\bar{\Psi} T_{a} \frac{\vec{\delta}}{\delta \bar{\Psi}} \Gamma+\Gamma \frac{\overleftarrow{\delta}}{\delta Y} T_{a} Y-\bar{Y} T_{a} \frac{\vec{\delta}}{\delta \bar{Y}} \Gamma\right\}
$$

In (2.33) the sum runs over all the fields which lie in the adjoint representation of $\mathrm{SU}(\mathrm{N})$,

$$
\Phi \in\left\{A_{\mu}, \rho_{\mu}, c, \sigma, \bar{c}, B\right\}
$$

In renormalized perturbation theory the Green functions of the nonabelian gauge theory are uniquely defined to all orders by the Ward identity of rigid SU(N)-invariance (2.32), the ST identity (2.29), by the linear gauge fixing function

$$
\left.\frac{\delta \Gamma}{\delta B_{a}}\right|_{\chi=0}=\xi B_{a}+\partial A_{a}
$$

and normalization conditions for fixing the remaining free parameters. As in QED we impose normalization conditions on the self-energies of vector bosons and fermions,

$$
\begin{array}{rlrl}
\Gamma_{A A}^{T}\left(p^{2}=0\right) & =0 & & \left.\frac{1}{p^{2}} \Gamma_{A A}^{T}\left(p^{2}\right)\right|_{p^{2}=\kappa^{2}}=1 \\
\left.\Gamma_{\bar{\Psi} \Psi}\right|_{p=M}=0 & & \left.\partial_{p} \Gamma_{\bar{\Psi} \Psi}\right|_{p=\kappa}=\mathbf{1} .
\end{array}
$$

Here $\Gamma_{A A}^{T}$ denotes the transversal part of the vector 2-point functions:

$$
\Gamma_{A_{a}^{\mu} A_{b}^{\nu}}=\delta_{a b}\left(\left(\eta^{\mu \nu}-\frac{p^{\mu} p^{\nu}}{p^{2}}\right) \Gamma_{A A}^{T}\left(p^{2}\right)+\frac{p^{\mu} p^{\nu}}{p^{2}} \Gamma_{A A}^{L}\left(p^{2}\right)\right)
$$

Since the ST identity does not explicitly depend on the coupling, these conditions have to be supplemented by a normalization condition for the coupling. However, having introduced the BRS-varying gauge parameter, it turns out that there remains one invariant counterterm to the coupling, which has to be independent of the gauge parameter. Gauge parameter dependence of the coupling counterterm is completely determined by the $\chi$-enlarged ST identity. If one uses for QCD an invariant scheme like dimensional regularization this requirement can be fulfilled by adjusting only gauge parameter independent counterterms to the gauge coupling. This is the case for example in the MS and $\overline{\mathrm{MS}}$ scheme, but not in the momentum subtraction scheme introduced in [10]. If one wants to define the coupling in a scheme-independent way at a specific normalization point, one has to introduce a normalization value $\xi_{o}$ [2] of the gauge parameter $\xi$, at which the coupling is normalized,

$$
\left.\Gamma_{A_{a \mu} \bar{\Psi} \Psi}\right|_{\substack{p_{s y m}=\kappa \\ \xi=\xi_{o}}}=g T_{a} \gamma^{\mu}
$$

or an equivalent normalization condition imposed on the vector boson vertex. Here $p_{\text {sym }}$ denotes the symmetric momentum:

$$
p_{i}^{2}=p^{2} \quad p_{i} p_{j}=-\frac{1}{3} p^{2} \quad \text { if } \quad i \neq j
$$


In fact, having a look to explicit expressions [10, 11] it is seen that this requirement is far from being trivial. The local part of the 3-point vector vertex functions, for example, depends on the gauge parameter through the power $\xi^{3}$ and this dependence cannot be removed by the normalization condition according to the above construction. (Another example, how $\xi_{0}$ enters the vertex functions, is provided by the background field gauge and presented in (5.22) of this paper.)

The important point in the construction of nonabelian gauge theories, when using a BRSvarying gauge parameter $\xi$, is that one is able to prove gauge parameter independence of the gauge $\beta$-functions of the CS equation and RG equation to all orders of perturbation theory independently of a specific scheme, and finally one can proceed to prove $\xi$-independence of Green functions of invariant operators. In addition to the CS equation

$$
\begin{aligned}
\left(\kappa \partial_{\kappa}+\right. & M \partial_{M}+\beta_{g} g \partial_{g}-\gamma_{A}\left(\int \mathrm{d}^{4} x\left(A_{a} \frac{\delta}{\delta A_{a}}-B_{a} \frac{\delta}{\delta B_{a}}-\bar{c}_{a} \frac{\delta}{\delta \bar{c}_{a}}\right)+2 \xi \partial_{\xi}\right) \\
& \left.-\gamma_{f} \int \mathrm{d}^{4} x\left(\bar{\Psi} \frac{\vec{\delta}}{\delta \bar{\Psi}}+\frac{\overleftarrow{\delta}}{\delta \Psi} \Psi\right)-\gamma_{c} \int \mathrm{d}^{4} x\left(c_{a} \frac{\delta}{\delta c_{a}}\right)\right)\left.\Gamma\right|_{\substack{e x t . f .=0 \\
\chi=0}}=\left[\Delta_{m}\right]_{3}^{3} \cdot \Gamma
\end{aligned}
$$

and the RG equation

$$
\begin{aligned}
\left(\kappa \partial_{\kappa}+\tilde{\beta}_{g} g \partial_{g}\right. & -\tilde{\gamma}_{A}\left(\int \mathrm{d}^{4} x\left(A_{a} \frac{\delta}{\delta A_{a}}-B_{a} \frac{\delta}{\delta B_{a}}-\bar{c}_{a} \frac{\delta}{\delta \bar{c}_{a}}\right)+2 \xi \partial_{\xi}\right) \\
& \left.-\tilde{\gamma}_{f} \int \mathrm{d}^{4} x\left(\bar{\Psi} \frac{\vec{\delta}}{\delta \bar{\Psi}}+\frac{\overleftarrow{\delta}}{\delta \Psi} \Psi\right)-\tilde{\gamma}_{c} \int \mathrm{d}^{4} x\left(c_{a} \frac{\delta}{\delta c_{a}}\right)\right)\left.\Gamma\right|_{\substack{e x t . f .=0 \\
x=0}}=0
\end{aligned}
$$

there is an additional differential equation, which governs the dependence on the normalization value $\xi_{o}$ of the gauge parameter $\xi[2]$ :

$$
\left(\xi_{o} \partial_{\xi_{o}}+\beta_{g}^{\xi_{0}} g \partial_{g}\right) \Gamma=0
$$

with

$$
\partial_{\xi} \beta_{g}=0 \quad \partial_{\xi} \tilde{\beta}_{g}=0 \quad \partial_{\xi} \beta_{g}^{\xi_{0}}=0
$$

to all orders. Of course, all these $\beta$-functions are scheme-dependent in higher orders, in particular they depend on $\kappa, M$ and $\xi_{o}$.

Due to the fact that there does not exist a gauge Ward identity the anomalous dimensions for the vector boson self energies $\gamma_{A}$ and $\tilde{\gamma}_{A}$ are not related to the $\beta$-functions of the coupling. The invariant charge is the solution of the homogeneous RG equation and of the characteristic equations,

$$
\left(\kappa \partial_{\kappa}+\beta_{g} g \partial_{g}\right) Q_{g}\left(p^{2}, \kappa^{2}, \xi_{0}\right)=0 \quad \text { with }\left.\quad Q_{g}\left(p^{2}, \kappa^{2}, \xi_{0}\right)\right|_{p^{2}=\kappa^{2}}=g^{2}
$$


and hence it cannot be related to a combination of vector 2-point functions. It has been proposed by the Pinch technique (PT) approach to nonabelian gauge theories 《7, \& to construct effective Green functions, which satisfy a nonabelian gauge Ward identity. Furthermore, among other requirements, it is claimed that the PT 2-point functions are indeed gauge parameter independent objects and satisfy the RG equation of the invariant charge in the asymptotic region. Although the PT approach looks promising, it is nevertheless difficult for an abstract analysis because it cannot be simply translated into the language of usual perturbation theory using a classical action and Feynman rules for systematically calculating higher order corrections.

Another way to arrive at nonabelian Ward identities has been provided by the background field gauge [3, 4]. It has been pointed out, that in the Feynman gauge the Green functions of the background fields indeed coincide with the ones of the PT [5, 12]. However the 2point background field functions depend on the gauge parameter due to local contributions in 1-loop order. In the present paper we show by the algebraic method using the BRSvarying gauge parameter that the $\xi$-dependent contributions indeed arise from the fact that the counterterms to the coupling cannot be adjusted arbitrarily concerning their gauge parameter dependence. In the explicit calculations these properties are ensured by using the MS scheme instead of definite normalization conditions. Hence the 2-point background field function is not an appropriate object for defining a RG-invariant charge. However, finally we will show that it is possible to modify the background gauge Ward identity in such a way, that a certain object constructed with the help of the background 2-point functions is gauge parameter independent and satisfies the homogeneous RG equation. The usual background Ward identity will only be valid at the normalization value $\xi_{0}$ of the gauge parameter $\xi$ (and at $\chi=0$ ). We explicitly construct this invariant charge up to 3-loop order and show that this construction is unique. Since we had to introduce the gauge parameter normalization $\xi_{o}$ we also have to consider the $\xi_{o}$-dependence of the invariant charge. We claim, that the invariant charge should be $\xi_{o}$-independent in the same way as it is independent of the normalization point $\kappa$ by fulfilling the homogeneous $\xi_{o}$-equation. However, when we apply the $\xi_{o}$-equation on the RG-invariant combination of 2-point functions it turns out, that this combination does not satisfy the homogeneous $\xi_{o}$-equation, and in this way the constructed object fails to be an invariant charge of QCD. 


\section{The tree approximation and the current construction of background field gauge}

Looking for a systematic definition of the pinch technique in higher orders, the current algebra approach to the pinch technique [8] seems us to be the most promising approach. Furthermore, in our opinion it is quite related to the background gauge field due to the following reason: If one wants to construct insertions of gauge currents into Green functions systematically in quantum field theory one necessarily ends up with the background field gauge, where one understands the background field as an external vector field coupled to the gauge current. In this section we want to present the current construction of background field gauge, and, at the same time, we want to fix our notations and conventions in the tree approximation.

Having constructed the Green functions of nonabelian gauge theories satisfying the ST identity and the Ward identity, we use from the action principle that gauge invariance is broken by insertions, which are $\mathrm{s}_{\Gamma}$-invariants:

$$
\mathbf{w}_{a} \Gamma=\left[\partial^{\mu} J_{a \mu}\right]_{4}^{4} \cdot \Gamma
$$

with

$$
\mathrm{s}_{\Gamma}\left(\left[\partial^{\mu} J_{a \mu}\right]_{4}^{4} \cdot \Gamma\right)=0
$$

Here $\mathbf{w}_{a}$ is the non-integrated version of the rigid Ward operator (2.33),

$$
\mathbf{w}_{a} \Gamma \equiv-\sum_{\Phi} f_{a b c} \Phi_{b} \frac{\delta \Gamma}{\delta \Phi_{c}}+\Gamma \frac{\overleftarrow{\delta}}{\delta \Psi} T_{a} \Psi-\bar{\Psi} T_{a} \frac{\vec{\delta}}{\delta \bar{\Psi}} \Gamma+\Gamma \frac{\overleftarrow{\delta}}{\delta Y} T_{a} Y-\bar{Y} T_{a} \frac{\vec{\delta}}{\delta \bar{Y}} \Gamma
$$

and $\mathrm{s}_{\Gamma}$ denotes the linearized version of the ST operator.

In the classical approximation all the breakings are local field polynomials, and one finds that all $\mathrm{s}_{\Gamma_{c l}}$-invariants are themselves $\mathrm{s}_{\Gamma_{c l}}$-variations:

$$
g \mathbf{w}_{a} \Gamma_{c l}=\mathrm{S}_{\Gamma_{c l}} \partial^{\mu}\left(\rho_{a \mu}+D_{\mu} \bar{c}_{a}\right)
$$

$D^{\mu}$ is the covariant derivative of the adjoint representation:

$$
D^{\mu} \Phi_{a}=\partial^{\mu} \Phi_{a}-g f_{a b c} \Phi_{b} A_{c}^{\mu} \quad \Phi \in\{c, \bar{c}, B\}
$$

Explicitly we arrive at:

$$
g \mathbf{w}_{a} \Gamma_{c l}=\partial^{\mu} \frac{\delta \Gamma_{c l}}{\delta A_{a}^{\mu}}+\partial^{\mu}\left(D_{\mu} B_{a}+g f_{a b c} \bar{c}_{b} D_{\mu} c_{c}\right) \equiv \partial^{\mu} \frac{\delta \Gamma_{c l}}{\delta A_{a}^{\mu}}+\partial^{\mu} \tilde{J}_{a \mu}
$$

From (3.6) we read off, that the nonabelian gauge Ward identity is broken by $B$-field and

ghost-field contributions. Since the latter ones are non-linear in the propagating fields 
this breaking becomes a non-trivial insertion in higher orders. Therefore, in order to be able to construct insertions of conserved currents in BRS-invariant gauge theories, we are going to couple the BRS-invariant current $\tilde{J}_{a}^{\mu}$ to a set of further external vector fields, which turn out to be the background fields $V_{a \mu}(x)$ :

$$
\Gamma_{c l} \longrightarrow \Gamma_{c l}+\int \mathrm{d}^{4} x \tilde{J}_{a}^{\mu} V_{a \mu}
$$

Since the background fields couple to a BRS-variation they are transformed under BRS into another set of external fields $C_{a}^{\mu}$ with ghost number 1 [13],

$$
\mathrm{s} V_{a \mu}=C_{a \mu} \quad, \quad \mathrm{s} C_{a \mu}=0
$$

By construction we end up with the usual tree action of background field gauges:

$$
\Gamma_{c l}=\Gamma_{Y M}+\Gamma_{\text {matter }}+\Gamma_{g . f .}^{B F}+\Gamma_{g h o s t}^{B F}
$$

with

$$
\begin{aligned}
\Gamma_{\text {g.f. }}^{B F} & =\int \mathrm{d}^{4} x\left\{\frac{\xi}{2} B_{a} B_{a}+B_{a}\left(\partial A_{a}-\partial V_{a}\right)-g f_{a b c} B_{a} A_{b} V_{c}\right\} \\
\Gamma_{\text {ghost }}^{B F} & =\int \mathrm{d}^{4} x\left\{\frac{1}{2} \chi \bar{c}_{a} B_{a}-\bar{c}_{a} D^{V} D^{A} c_{a}+\bar{c}_{a} D^{A} C_{a}\right\}
\end{aligned}
$$

Here $D^{V}$ and $D^{A}$ denote the covariant derivatives with respect to vectors (3.5) and background vectors:

$$
D_{\mu}^{V} \Phi_{a}=\partial_{\mu} \Phi_{a}-g f_{a b c} \Phi_{b} V_{c \mu}
$$

The action (3.9) is invariant under the enlarged Slavnov-Taylor identity,

$$
\mathcal{S}\left(\Gamma_{c l}\right)=0
$$

with

$\mathcal{S}(\Gamma) \equiv \int \mathrm{d}^{4} x\left\{\frac{\delta \Gamma}{\delta \rho_{a}^{\mu}} \frac{\delta \Gamma}{\delta A_{a \mu}}+\frac{\delta \Gamma}{\delta \sigma_{a}} \frac{\delta \Gamma}{\delta c_{a}}+B_{a} \frac{\delta \Gamma}{\delta \bar{c}_{a}}+C_{a}^{\mu} \frac{\delta \Gamma}{\delta V_{a}^{\mu}}+\Gamma \frac{\overleftarrow{\delta}}{\delta \Psi} \frac{\vec{\delta}}{\delta \bar{Y}} \Gamma+\Gamma \frac{\overleftarrow{\delta}}{\delta Y} \frac{\vec{\delta}}{\delta \bar{\Psi}} \Gamma\right\}+\chi \partial_{\xi} \Gamma$

and satisfies the local gauge Ward identity:

$$
\left(g \mathbf{w}_{a}-\partial^{\nu} \frac{\delta}{\delta A_{a}^{\nu}}-\partial^{\nu} \frac{\delta}{\delta V_{a}^{\nu}}\right) \Gamma_{c l}=0
$$

$\mathbf{w}_{a}$ is given in (3.3), but the sum over $\Phi$ now includes also the background fields $V_{a \mu}$ and their BRS transformations $C_{a \mu}$ :

$$
\Phi \in\left\{A_{\mu}, \rho_{\mu}, V_{\mu}, C_{\mu}, c, \sigma, \bar{c}, B\right\}
$$


In 113 the ST identity (3.13) and the Ward identity (3.14) have been considered for $\chi=0$ as the defining symmetries in the procedure of algebraic renormalization of the background field gauge. According to the fact, that we do not only want to construct background field Green functions but also a gauge parameter independent object out of 2-point functions we have to modify the local Ward identity in higher orders, but we will refer to [13] as far as possible.

The point, where the construction of the present paper differs from the usual background field method can be seen already from our approach to the tree action. Considering eq. (3.4) it is seen immediately, that it is by no means unique how to couple the gauge current to the vector fields $A_{a}^{\mu}$ and $V_{a}^{\mu}$. In the tree approximation different parametrizations are only trivial field redefinitions of the form

$$
A_{a}^{\mu} \longrightarrow A_{a}^{\mu}+\bar{z} V_{a}^{\mu}
$$

In higher orders, however, this freedom becomes an important ingredient of the construction when we want to fix the background 2-point function in such a way that we arrive at a gauge parameter independent combination to all orders.

\section{Normalization conditions and local invariants}

Having defined the tree approximation, the Green functions in renormalized perturbation theory are constructed according to a renormalization scheme, defining symmetries and normalization conditions. Due to the action principle the symmetries of lowest order are in 1-loop order at most broken by local polynomials:

$$
\begin{aligned}
\mathcal{S}\left(\Gamma^{R}\right) & =\Delta_{b r s}+O\left(\hbar^{2}\right) \\
\left(g \mathbf{w}_{a}-\partial \frac{\delta}{\delta A_{a}}-\partial \frac{\delta}{\delta V_{a}}\right) \Gamma^{R} & =\partial J_{a}+O\left(\hbar^{2}\right)
\end{aligned}
$$

$\Gamma^{R}$ denotes the generating functional of subtracted finite 1PI Green functions to be calculated in a specific renormalization scheme such as the momentum subtraction scheme of BPHZL or using dimensional regularization with MS-subtraction of poles.

The breakings of the ST identity are restricted according to algebraic consistency by:

$$
\mathrm{S}_{\Gamma_{c l}} \Delta_{b r s}=0
$$

In [13] it has been shown that all possible breakings of the ST identity for $\chi=0$ have to

be BRS variations, if parity is conserved. The generalization to $\chi \neq 0$ is straightforward 
and proceeds along the lines of [2]:

$$
\Delta_{b r s}=-\mathrm{S}_{\Gamma_{c l}} \Gamma_{b r e a k}
$$

$\Gamma_{\text {break }}$ has the same quantum numbers as the classical action. For this reason we are able to absorb $\Delta_{b r s}$ into noninvariant counterterms to the classical action:

$$
\mathcal{S}\left(\Gamma^{R}+\Gamma_{\text {break }}\right)=O\left(\hbar^{2}\right)
$$

These counterterms are scheme-dependent and vanish immediately in an invariant scheme. Furthermore, it is clear that the proof outlined above can be extended to all orders of perturbation theory by induction in the loop expansion.

Hence, for proceeding, we will now assume that the ST identity has been established to all orders by an appropriate adjusting of counterterms. It then remains to find the free parameters of the model, i.e. the invariant counterterms $\Gamma_{i n v}$ carrying the quantum numbers of the action and obeying

$$
\mathrm{S}_{\Gamma_{c l}} \Gamma_{i n v}=0
$$

They have to be fixed by normalization conditions and/or the local Ward identity. Determining the general classical solution $\Gamma_{c l}^{g e n}$ of

$$
\mathcal{S}\left(\Gamma_{c l}^{g e n}\right)=0 \quad \text { with } \quad \operatorname{dim}^{U V} \Gamma_{c l}^{g e n} \leq 4
$$

allows to find all the invariant counterterms of higher orders. We do not want to give the details of the calculation here, because this calculation follows the lines of [2] (concerning gauge parameter dependence) and [13] (concerning the ST identity with background field).

The most general solution $\Gamma_{c l}^{g e n}$ can be decomposed, just as in the tree approximation, into the Yang-Mills part, the matter part and the external field part. We further split off the gauge fixing and ghost part, the latter being later on trivially determined from the ghost equation of motion:

$$
\Gamma_{c l}^{g e n}=\Gamma_{Y M}^{g e n}+\Gamma_{m a t t e r}^{g e n}+\Gamma_{e x t . f .}^{g e n}+\Gamma_{g . f .}^{g e n}+\Gamma_{\phi \pi}^{g e n}
$$

We find that the YM-part and the matter part depend on the vector fields only via the combination

$$
A_{\mu}^{o}=z_{A} A^{\mu}+z_{V} V^{\mu}
$$

where $z_{A}$ and $z_{V}$ are arbitrary parameters:

$$
\Gamma_{Y M}^{g e n}\left(A^{o}\right)=-\frac{1}{4} \int \mathrm{d}^{4} x G_{a}^{\mu \nu}\left(A^{o}\right) G_{a \mu \nu}\left(A^{o}\right)
$$


with

$$
G_{a}^{\mu \nu}\left(A^{o}\right) \equiv \partial^{\mu} A_{a}^{o \nu}-\partial^{\nu} A_{a}^{o \mu}+g^{o} f_{a b c} A_{b}^{o \mu} A_{c}^{o \nu}
$$

Introducing the bare fermion fields

$$
\Psi^{o}=z_{F} \Psi
$$

also the matter part takes its usual covariant form:

$$
\Gamma_{\text {matter }}^{\text {gen }}=\int \mathrm{d}^{4} x\left\{i \overline{\Psi^{o}} \gamma^{\mu} \partial_{\mu} \Psi^{o}+i g^{o} \overline{\Psi^{o}} \gamma^{\mu} A_{a \mu}^{o} T_{a} \Psi^{o}-M^{o} \overline{\Psi^{o}} \Psi^{o}\right\}
$$

The external field part does not only depend on $A^{o}$ but shows an explicit dependence on the background fields $V_{a}^{\mu}$ and their BRS transformations $C_{a}^{\mu}$ :

$$
\begin{aligned}
\Gamma_{\text {ext.f. }}^{\text {gen }}=\int \mathrm{d}^{4} x & \left\{-\frac{1}{2} f_{a b c} z_{G} g^{o} \sigma_{a} c_{b} c_{c}-\chi \partial_{\xi} \ln z_{G} \sigma_{a} c_{a}\right. \\
& +z_{A}^{-1} \rho_{a}^{\mu}\left(z_{G} \partial_{\mu} c_{a}+g^{o} z_{G} f_{a b c} A_{b \mu}^{o} c_{c}-z_{V} C_{a \mu}\right) \\
& +\chi z_{A}^{-1} \partial_{\xi} \ln z_{A} \rho_{a}^{\mu} A_{a \mu}^{o}+\chi z_{V} z_{A}^{-1} \partial_{\xi} \ln \frac{z_{V}}{z_{A}} \rho_{a}^{\mu} V_{a \mu} \\
& \left.-g^{o}\left(\bar{Y} T_{a} \Psi+\bar{\Psi} T_{a} Y\right) c_{a} z_{G}+\chi \partial_{\xi} \ln z_{F}(\bar{Y} \Psi-\bar{\Psi} Y)\right\}
\end{aligned}
$$

Having introduced a BRS-transforming gauge parameter, it is also $\chi$-dependent. The most remarkable point in the analysis is, however, the fact that both the parameter $g^{o}$, which is usually identified with the coupling, and the parameter $M^{o}$, which represents the bare mass of fermions, have to be gauge parameter independent in order to be consistent with the $\chi$-enlarged Slavnov-Taylor identity:

$$
\partial_{\xi} g^{o}=0 \quad \partial_{\xi} M^{o}=0
$$

The wave function renormalizations $z_{A}, z_{V}$ and $z_{F}$, on the other hand, are allowed to depend on $\xi$ arbitrarily.

Before proceeding to the gauge fixing sector we apply the general local abelian Ward operator, compatible with the algebra, to the general YM, matter and external field part and find by direct computation:

$$
\left.\left(g^{o}\left(z_{A} \bar{z}+z_{V}\right) \mathbf{w}_{a}-\bar{z} \partial \frac{\delta}{\delta A_{a}}-\partial \frac{\delta}{\delta V_{a}}\right)\left(\Gamma_{Y M}^{g e n}+\Gamma_{\text {matter }}^{g e n}+\Gamma_{\text {ext.f. }}^{g e n}\right)\right|_{\chi=0}=0
$$

$\bar{z}$ is a further by now undetermined parameter. Acting with this Ward operator on the general linear gauge fixing function which takes for $V=0$ the usual form,

$$
\left.\Gamma_{g . f}\right|_{V=0}=\int \mathrm{d}^{4} x\left\{\frac{\xi}{2} B_{a} B_{a}+B_{a} \partial A_{a}\right\},
$$


it turns out that the background field dependent part is uniquely determined from invariance with respect to the local Ward operator:

$$
\Gamma_{g . f}=\int \mathrm{d}^{4} x\left\{\frac{\xi}{2} B_{a} B_{a}+B_{a} \partial A_{a}-\bar{z} B_{a} \partial V_{a}-g^{o}\left(z_{A} \bar{z}+z_{V}\right) f_{a b c} B_{a} A_{b}^{\mu} V_{c \mu}\right\}
$$

The ghost part of the action is then uniquely given by integration of the ghost equation of motion and is by construction invariant under the local Ward identity:

$$
\begin{aligned}
-\frac{\delta}{\delta \bar{c}_{a}} \Gamma_{c l}^{g e n}= & \left(\partial^{\mu} \delta_{a c}+g^{o}\left(z_{A} \bar{z}+z_{V}\right) f_{a b c} V_{b}^{\mu}\right) \frac{\delta \Gamma_{c l}^{g e n}}{\delta \rho_{c}^{\mu}}-\left(\partial^{\mu} \delta_{a c} \bar{z}+g^{o}\left(z_{A} \bar{z}+z_{V}\right) f_{a b c} A_{b}^{\mu}\right) C_{c \mu} \\
& +\frac{1}{2} \chi B_{a}-\chi \partial_{\xi} \bar{z} \partial^{\mu} V_{a \mu}-\chi g^{o} \partial_{\xi}\left(z_{A} \bar{z}+z_{V}\right) f_{a b c} A_{b}^{\mu} V_{c \mu}
\end{aligned}
$$

Hence we have

$$
\Gamma_{c l}^{g e n}=\Gamma_{Y M}^{g e n}+\Gamma_{m a t t e r}^{g e n}+\Gamma_{e x t . f .}^{g e n}+\Gamma_{g . f .}+\Gamma_{\phi \pi}^{g e n},
$$

and the five parts of the general classical action are given in (4.9), (4.12), (4.13), (4.17) and (4.18), respectively.

Finally taking into account $\chi$-dependent contributions to the local WI, these contributions being uniquely constructed by requiring the Ward operator to be a $\mathrm{s}_{\Gamma}$-invariant operator, we end up with the following local WI:

$$
\left(g^{o}\left(z_{A} \bar{z}+z_{V}\right) \mathbf{w}_{a}-\bar{z} \partial \frac{\delta}{\delta A_{a}}-\partial \frac{\delta}{\delta V_{a}}\right) \Gamma_{c l}^{g e n}=\chi \partial^{\mu}\left(\partial_{\xi} \bar{z} \rho_{a \mu}-\partial_{\xi} \ln \left(z_{A} \bar{z}+z_{V}\right)\left(\frac{\delta \Gamma_{c l}^{g e n}}{\delta C_{a}^{\mu}}+\bar{z} \rho_{a \mu}\right)\right)
$$

The local WI does not impose any further restrictions on the $\xi$-dependence of the free parameters of the theory.

We now want to fix the free parameters by normalization conditions. For the vector boson and fermion self-energies we take the usual QCD normalization conditions as given in (2.36), (2.37). These normalization conditions fix $z_{A}, z_{F}$ and $M^{o}$. On-shell normalization conditions have been shown to be in agreement with the ST identity without being forced to introduce a normalization value $\xi_{o}$ of the gauge parameter $\xi$ into these normalization conditions [14]. Therefore it remains to fix the parameters $\bar{z}, z_{V}$ and $g^{o}$. In a first step, we eliminate one parameter from the gauge fixing function by requiring the gauge fixing to be

$$
\left.\frac{\delta \Gamma}{\delta B_{a}}\right|_{\chi=0}=\xi B_{a}+\partial A_{a}-\bar{z} \partial V_{a}-g f_{a b c} A_{b} V_{c}
$$

$g$ is the coupling of the tree approximation. Hence we have:

$$
g^{o}\left(z_{A} \bar{z}+z_{V}\right)=g \quad \text { and } \quad \partial_{\xi}\left(z_{A} \bar{z}\right)=-\partial_{\xi} z_{V}
$$

The coupling constant is fixed by the Ward identity and we remain with the 2 free parameters $z_{V}$ and $\bar{z}$. Due to gauge parameter independence of the coupling (4.14) gauge 
parameter dependence of $z_{V}$ and $\bar{z}$ is related to the $\xi$-dependence of $z_{A}$. Therefore not all of these three parameters can be fixed arbitrarily concerning their gauge parameter dependence. $z_{A}$ has already been fixed by the normalization of the vector boson residuum (2.36). In the usual background field approach one chooses $\bar{z}$ to take a definite value such as for example $\bar{z}=1$ and disposes in this way on the gauge parameter dependence of $\bar{z}$. The gauge parameter dependence of $z_{V}$ is then restricted and the background vector boson 2-point function can only be normalized concerning its gauge parameter independent part. (See (5.20), (5.21) and (5.22) for the respective Ward identity, the normalization condition and the explicit expression.) For this reason the 2-point function in the usual background field gauge depends on the gauge parameter $\xi$. One can, however, also proceed differently (having at our disposal the additional parameter $\bar{z}$ ) and dispose on $z_{V}$ completely by a normalization on the background vectors (see (5.13)). But then the gauge parameter dependent part of $\bar{z}$ is completely determined by the ST identity, i.e. by (4.22), and can be fixed only at $\xi=\xi_{o}$. (See (5.15) and (5.18), (5.19) for the generalized Ward identity and explicit expressions.)

In the next section we will establish the local Ward identity in higher orders. There we will show that all the breakings of the local Ward identity can be absorbed by adjusting the free parameters of the invariant counterterms, which we have computed in the general classical approximation.

\section{The local Ward identity in higher orders}

In order to prove the local WI in higher orders we start from the renormalized STsymmetric Green functions as constructed in (4.4):

$$
\mathcal{S}\left(\Gamma_{\text {sym }}^{R}\right)=0+O\left(\hbar^{2}\right) \quad \text { with } \quad\left(\Gamma_{\text {sym }}^{R}\right)^{(\leq 1)}=\left(\Gamma^{R}\right)^{(\leq 1)}+\Gamma_{\text {break }}^{(1)}
$$

As for the ST identity we will proceed by induction in the loop expansion and give explicitly the step from the tree approximation to 1-loop order.

Furthermore we postulate the linear gauge fixing function of the tree approximation to be valid for $\Gamma_{\text {sym }}^{R}$ :

$$
\left.\frac{\delta \Gamma_{\text {sym }}^{R}}{\delta B_{a}}\right|_{\chi=0}=\xi B_{a}+\partial A_{a}-\tilde{z} \partial V_{a}-g f_{a b c} A_{b} V_{c}
$$

On the gauge condition we fix the normalization of the $B_{a}$-field, of the gauge parameter and of the background field $V_{a}^{\mu}$. As long as a Ward identity is not used $\tilde{z}$ is a free parameter of the gauge condition. One has also to note that the field normalization $z_{V}$ 
(4.8), which gives the mixing between the propagating field $A_{a}^{\mu}$ and the background field $V_{a}^{\mu}$, is not fixed on the gauge condition without considering a Ward identity. According to the discussion of the last section the Green functions $\Gamma_{s y m}^{R}$ are not uniquely determined by the subtraction scheme, the Slavnov-Taylor identity and by requiring the linear gauge fixing, but they can be modified by the invariant counterterms, which are related to the free parameters of the general classical solution (cf. (4.19)). In perturbation theory these invariant counterterms are most easily expressed in terms of invariant operators acting on the classical action [13],

$$
\begin{aligned}
\Gamma^{(\leq 1)}=\left(\Gamma_{s y m}^{R}\right)^{(\leq 1)} & +\delta z_{A}^{(1)}\left(\int \mathrm{d}^{4} x\left(A_{a \mu} \frac{\delta}{\delta A_{a \mu}}-\rho_{a \mu} \frac{\delta}{\delta \rho_{a \mu}}-B_{a} \frac{\delta}{\delta B_{a}}-\bar{c}_{a} \frac{\delta}{\delta \bar{c}_{a}}\right)+2 \xi \partial_{\xi}+2 \chi \partial_{\chi}\right) \Gamma_{c l} \\
& +\chi \partial_{\xi} \delta z_{A}^{(1)}\left(\int \mathrm{d}^{4} x\left(\rho_{a \mu} A_{a}^{\mu}-\bar{c}_{a} \frac{\delta \Gamma_{c l}}{\delta B_{a}}\right)+2 \xi \partial_{\chi} \Gamma_{c l}\right) \\
& +\delta z_{G}^{(1)} \int \mathrm{d}^{4} x\left(c_{a} \frac{\delta}{\delta c_{a}}-\sigma_{a} \frac{\delta}{\delta \sigma_{a}}\right) \Gamma_{c l}-\chi \partial_{\xi} \delta z_{G}^{(1)} \int \mathrm{d}^{4} x \sigma_{a} c_{a} \\
& +\delta z_{V}^{(1)} \int \mathrm{d}^{4} x\left(V_{a \mu} \frac{\delta \Gamma_{c l}}{\delta A_{a \mu}}-\rho_{a \mu} C_{a}^{\mu}\right)+\chi \partial_{\xi} \delta z_{V}^{(1)} \int \mathrm{d}^{4} x \rho_{a \mu} V_{a}^{\mu} \\
& +\delta z_{F}^{(1)} \int \mathrm{d}^{4} x\left(\bar{\Psi} \frac{\vec{\delta}}{\delta \bar{\Psi}}+\frac{\overleftarrow{\delta}}{\delta \Psi} \Psi \bar{Y} \frac{\vec{\delta}}{\delta \bar{Y}}-\frac{\overleftarrow{\delta}}{\delta Y} Y\right) \Gamma_{c l} \\
& +\chi \partial_{\xi} \delta z_{F}^{(1)} \int \mathrm{d}^{4} x(\bar{Y} \Psi-\bar{\Psi} Y) \\
& +\delta g^{(1)}\left(g \partial_{g}-\int \mathrm{d}^{4} x\left(V_{a \mu} \frac{\delta}{\delta V_{a \mu}}+C_{a \mu} \frac{\delta}{\delta C_{a \mu}}\right)\right) \Gamma_{c l}+\delta M^{(1)} M \partial_{M} \Gamma_{c l}
\end{aligned}
$$

with:

$$
\mathcal{S}(\Gamma)=\mathcal{S}\left(\Gamma_{\text {sym }}^{R}\right)=0+O\left(\hbar^{2}\right)
$$

According to (4.14) the counterterms proportional to $\delta g^{(1)}$ and $\delta M^{(1)}$ have to be gauge parameter independent:

$$
\partial_{\xi} \delta g^{(1)}=0 \quad \partial_{\xi} \delta M^{(1)}=0
$$

The counterterms to the gauge fixing function are already constrained in such a way that $\Gamma$ satisfies the linear gauge condition (5.2):

$$
\left.\frac{\delta \Gamma}{\delta B_{a}}\right|_{\chi=0}=\xi B_{a}+\partial A_{a}-\left(1+\delta \tilde{z}^{(1)}-\delta z_{A}^{(1)}-\delta z_{V}^{(1)}-\delta g^{(1)}\right) \partial V_{a}-g f_{a b c} A_{b} V_{c}
$$

By now all the free parameters can be fixed by independent normalization conditions.

In a first step we apply the local Ward operator of the tree approximation to $\Gamma_{\text {sym }}^{R}$. According to the action principle this WI is broken by local contributions $\partial^{\mu} J_{a \mu}^{(1)}$ with $\phi \pi$-charge 0 and dimension bounded by 4 :

$$
\left(g \mathbf{w}_{a}-\partial \frac{\delta}{\delta A_{a}}-\partial \frac{\delta}{\delta V_{a}}\right) \Gamma_{s y m}^{R}=\partial^{\mu} J_{a \mu}^{(1)}+O\left(\hbar^{2}\right)
$$


Since the 1PI Green functions $\Gamma_{\text {sym }}^{R}$ are assumed to satisfy the ST identity (5.1), the breakings $\partial^{\mu} J_{a \mu}^{(1)}$ have also to be $\mathrm{s}_{\Gamma_{c l}}$-invariant:

$$
\mathrm{s}_{\Gamma_{c l}} \partial J_{a}^{(1)}=0
$$

It turns out that the most general expression for the current $J_{a}^{\mu}$ is a $\mathrm{S}_{\Gamma_{c l}}$-variation:

$$
\begin{aligned}
J_{a}^{\mu} & =\mathrm{s}_{\Gamma_{c l}}\left(u_{1}(\xi) \rho_{a}^{\mu}+u_{2}(\xi) \frac{\delta \Gamma_{c l}}{\delta C_{a \mu}}+u_{3}(\xi) \partial^{\mu} \bar{c}_{a}\right) \\
& =u_{1} \frac{\delta \Gamma_{c l}}{\delta A_{a \mu}}+u_{2} \frac{\delta \Gamma_{c l}}{\delta V_{a \mu}}+u_{3} \partial^{\mu} B_{a}+\chi \partial_{\xi} u_{1} \rho_{a}^{\mu}+\chi \partial_{\xi} u_{2} \frac{\delta \Gamma_{c l}}{\delta C_{a \mu}}+\chi \partial_{\xi} u_{3} \partial^{\mu} \bar{c}_{a}
\end{aligned}
$$

Testing (5.7), (5.9) on the gauge condition (5.2) we find that the coefficients $u_{2}$ and $u_{3}$ vanish, whereas $u_{1}$ is arbitrary and determines the parameter $\tilde{z}=1+\delta \tilde{z}^{(1)}$ which appears in the gauge fixing condition (5.2):

$$
u_{2}=u_{3}=0 \quad \delta \tilde{z}^{(1)}=u_{1}
$$

Next we apply the Ward operator of the tree approximation to the general 1PI Green functions $\Gamma$ (5.3). Taking into account the results (5.9) and (5.10) we finally end up with:

$$
\begin{aligned}
& \left(g \mathbf{w}_{a}-\partial^{\mu} \frac{\delta}{\delta A_{a}^{\mu}}-\partial^{\mu} \frac{\delta}{\delta V_{a}^{\mu}}\right) \Gamma \\
= & \left(u_{1}^{(1)}-\delta z_{A}^{(1)}-\delta z_{V}^{(1)}\right) \partial^{\mu} \frac{\delta \Gamma_{c l}}{\delta A_{a}^{\mu}}+\chi \partial_{\xi}\left(u_{1}^{(1)}-\delta z_{A}^{(1)}-\delta z_{V}^{(1)}\right) \partial^{\mu} \rho_{a \mu} \\
& -\delta g^{(1)}\left(g \mathbf{w}_{a}-\partial^{\mu} \frac{\delta}{\delta V_{a}^{\mu}}\right) \Gamma_{c l}+O\left(\hbar^{2}\right) \\
= & \left(u_{1}^{(1)}-\delta z_{A}^{(1)}-\delta z_{V}^{(1)}-\delta g^{(1)}\right) \partial^{\mu} \frac{\delta \Gamma_{c l}}{\delta A_{a}^{\mu}}+\chi \partial_{\xi}\left(u_{1}^{(1)}-\delta z_{A}^{(1)}-\delta z_{V}^{(1)}\right) \partial^{\mu} \rho_{a \mu}+O\left(\hbar^{2}\right)
\end{aligned}
$$

Now we are able to follow the discussion of the last section: Having fixed the counterterm $\delta z_{A}^{(1)}$ by the normalization condition on the residuum of the transversal vector propagator $(\operatorname{see}(2.36))$,

$$
\left.\frac{1}{p^{2}} \Gamma_{A A}^{T}\left(p^{2}\right)\right|_{p^{2}=\kappa^{2}}=1
$$

and fixing the counterterm $\delta z_{V}^{(1)}$ by a gauge parameter independent normalization condition on the external vector bosonst,

$$
\left.\frac{1}{p^{2}}\left(\Gamma_{V V}^{T}+2 \Gamma_{V A}^{T}+\Gamma_{A A}^{T}\right)\right|_{p^{2}=\kappa^{2}}=1
$$

the local Ward identity of the tree approximation can only be established in 1-loop order concerning its $\xi$-independent part by requiring:

$$
u_{1}^{(1)}-\delta z_{A}^{(1)}-\left.\delta z_{V}^{(1)}\right|_{\xi=\xi_{o}}=\delta g^{(1)}
$$

\footnotetext{
${ }^{2} \Gamma_{A A}^{T}$ is defined in (2.38), and analogous expressions are valid for $\Gamma_{A V}^{T}$ and $\Gamma_{V V}^{T}$.
} 
The $\xi$-dependent part is completely determined by the $\chi$-enlarged Slavnov-Taylor identity. In other words: Using the normalization conditions (5.12) and (5.13), we are able to establish the following local WI in 1-loop order:

$$
\left(g \mathbf{w}_{a}-\bar{z} \partial \frac{\delta}{\delta A_{a}}-\partial \frac{\delta}{\delta V_{a}}\right) \Gamma=\chi \partial_{\xi} \bar{z} \partial \rho_{a}+O\left(\hbar^{2}\right)
$$

with

$$
\bar{z} \equiv 1+u^{(1)}-\delta z_{A}^{(1)}-\delta z_{V}^{(1)}-\delta g^{(1)}
$$

and

$$
\bar{z} \equiv \bar{z}\left(\xi, \xi_{o}\right) \quad \text { and }\left.\quad \bar{z}\right|_{\xi=\xi_{o}}=1
$$

At the normalization value $\xi_{0}$ of the gauge parameter $\xi$ and at $\chi=0$ the 1PI Green functions $\Gamma$ satisfy the Ward identity of the tree approximation:

$$
\left.\left(g \mathbf{w}_{a}-\partial \frac{\delta}{\delta A_{a}}-\partial \frac{\delta}{\delta V_{a}}\right) \Gamma\right|_{\substack{\xi=\xi_{o} \\ \chi=0}}=0
$$

In 1-loop order we find the following explicit expressions for the vector self-energies and the parameter $\bar{z}$ :

$$
\begin{aligned}
\Gamma_{A A}^{T(1)} & =\frac{g^{2}}{16 \pi^{2}}\left[\left(\frac{-13}{16+\frac{\xi}{2}}\right) N+\frac{2}{3} n_{f}\right] \ln \frac{p^{2}}{\kappa^{2}} \\
\Gamma_{A A}^{T(1)}+2 \Gamma_{V A}^{T(1)}+\Gamma_{V V}^{T(1)} & =\frac{g^{2}}{16 \pi^{2}}\left[\frac{-11}{3} N+\frac{2}{3} n_{f}\right] \ln \frac{p^{2}}{\kappa^{2}} \\
\delta \bar{z}^{(1)} & =\frac{g^{2}}{32 \pi^{2}}\left[\frac{(\xi-1)(\xi+7)}{4} N-\frac{\left(\xi_{o}-1\right)\left(\xi_{o}+7\right)}{4} N\right]
\end{aligned}
$$

In the above construction the relevance of algebraic gauge parameter control becomes quite striking: The arguments we have given here follow to some extent the arguments of [13] but differ in the conclusions. Having a $\xi_{o}$-independent normalization condition for $\delta z_{V}$ (5.13) and also for $\delta z_{A}$ (5.12) the Ward identity of the tree approximation can only be established at $\xi=\xi_{o}$ (and $\chi=0$ ) in higher orders. If one wants to establish the Ward identity of the tree approximation for all values of $\xi$ in higher orders, i.e. in 1-loop order

$$
\left(g \mathbf{w}_{a}-\partial \frac{\delta}{\delta A_{a}}-\partial \frac{\delta}{\delta V_{a}}\right) \Gamma=O\left(\hbar^{2}\right)
$$

the normalization condition for the background vector has to be stated at $\xi=\xi_{o}$ and, accordingly, (5.13) has to be modified to:

$$
\left.\frac{1}{p^{2}}\left(\Gamma_{V V}^{T}+2 \Gamma_{V A}^{T}+\Gamma_{A A}^{T}\right)\right|_{\substack{p^{2}=\kappa^{2} \\ \xi=\xi_{0}}}=1
$$


The local part of the combination $\Gamma_{V V}^{T}+2 \Gamma_{V A}^{T}+\Gamma_{A A}^{T}$ then becomes gauge parameter dependent and is determined by the Ward identity. Explicitly, in this case, we find the following gauge parameter dependent expression in 1-loop order:

$$
\begin{aligned}
\Gamma_{A A}^{T(1)}+2 \Gamma_{V A}^{T(1)}+\Gamma_{V V}^{T(1)}=\frac{g^{2}}{16 \pi^{2}} & {\left[\left(\frac{-11}{3} N+\frac{2}{3} n_{f}\right) \ln \frac{p^{2}}{\kappa^{2}}\right.} \\
& \left.+\frac{(\xi-1)(\xi+7)}{4} N-\frac{\left(\xi_{o}-1\right)\left(\xi_{o}+7\right)}{4} N\right]
\end{aligned}
$$

In a final step the Ward identities are established by induction to all orders. This is achieved by repeating the discussion given in 1-loop order. In the following sections we will show that we are now able to construct an RG-invariant object out of 2-point functions, which is gauge parameter independent and which, in lowest order, is related to the combination $\Gamma_{A A}^{T}+2 \Gamma_{V A}^{T}+\Gamma_{V V}^{T}$ (5.18) as suggested by the Pinch technique construction.

\section{Parametric differential equations}

In this section we derive the Callan-Symanzik equation of the nonabelian gauge theory in the generalized background field gauge. In addition we will derive the differential equations, which govern the dependence on the normalization points, namely the renormalization group equation and the $\xi_{o}$-equation. (In (2.41) - (2.43) we have given the respective equations in conventional linear gauges.) Having rigorously derived these differential equations we can systematically proceed to construct an invariant charge out of 2-point functions. According to the existence of a Ward identity it is seen, that there is a certain combination of 2-point functions, whose leading logarithms are given by the $\beta$-functions of the coupling constant. By introducing the BRS-varying gauge parameter and constructing Green functions invariant with respect to the $\chi$-enlarged ST identity, it is ensured, that $\beta$-functions are gauge parameter independent and so are the leading logarithms of these 2-point functions $([4,15])$. For this reason such an object seems to be an appropriate object for constructing a RG-invariant charge like in QED (see section 2, (2.15)-(2.17)). Due to the generalized background gauge fixing it is even possible to remove the local $\xi$-dependent parameters in 1-loop order (cf. (5.18)). Indeed we will be able to construct an object which satisfies the RG equation of the invariant charge up to 3-loop order (see section 7), but we will also find that this object does not satisfy the appropriate differential $\xi_{o}$-equation (see section 8).

In order to derive the parametric differential equations in question we first note that the corresponding differential operators $\kappa \partial_{\kappa}+M \partial_{M}$ (for CS), $\kappa \partial_{\kappa}$ (for RG) and $\xi_{0} \partial_{\xi_{0}}$ are BRS- 
symmetric and rigidly symmetric differential operators. Hence according to the quantum action principle

$$
\underline{\lambda} \partial_{\underline{\lambda}} \Gamma=\Delta_{\underline{\lambda}} \cdot \Gamma \text { for } \underline{\lambda}=\{\kappa, M\}, \kappa, \xi_{0}
$$

the r.h. sides $\Delta_{\underline{\lambda}} \cdot \Gamma$ have to be symmetric insertions,

$$
\mathrm{s}_{\Gamma}\left(\Delta_{\underline{\lambda}} \cdot \Gamma\right)=0 \quad \mathcal{W}_{a}\left(\Delta_{\underline{\lambda}} \cdot \Gamma\right)=0,
$$

of dimension bounded by 4 and with $\phi \pi$-charge 0 . A basis for these insertions has already been constructed in section 5 , see (5.3).

Therefore one immediately obtains for the CS equation:

$$
\begin{aligned}
& \left(\kappa \partial_{\kappa}+M \partial_{M}+\beta_{g}\left(g \partial_{g}-\int \mathrm{d}^{4} x\left(V_{a \mu} \frac{\delta}{\delta V_{a \mu}}+C_{a \mu} \frac{\delta}{\delta C_{a \mu}}\right)\right)\right. \\
& -\gamma_{A}\left(\int \mathrm{d}^{4} x\left(A_{a \mu} \frac{\delta}{\delta A_{a \mu}}-\rho_{a \mu} \frac{\delta}{\delta \rho_{a \mu}}-B_{a} \frac{\delta}{\delta B_{a}}-\bar{c}_{a} \frac{\delta}{\delta \bar{c}_{a}}\right)+2 \xi \partial_{\xi}+2 \chi \partial_{\chi}\right) \\
& -\gamma_{\bar{V}} \int \mathrm{d}^{4} x V_{a \mu} \frac{\delta}{\delta A_{a \mu}}-\gamma_{G} \int \mathrm{d}^{4} x\left(c_{a} \frac{\delta}{\delta c_{a}}-\sigma_{a} \frac{\delta}{\delta \sigma_{a}}\right) \\
& -\gamma_{F} \int \mathrm{d}^{4} x\left(\bar{\Psi} \frac{\vec{\delta}}{\delta \bar{\Psi}}+\frac{\overleftarrow{\delta}}{\delta \Psi} \Psi-\bar{Y} \frac{\vec{\delta}}{\delta \bar{Y}}-\frac{\overleftarrow{\delta}}{\delta Y} Y\right) \\
& \left.+\chi \partial_{\xi} \gamma_{A}\left(\int \mathrm{d}^{4} x\left(\bar{c}_{a} \frac{\delta}{\delta B_{a}}\right)-2 \xi \partial_{\chi}\right)\right) \Gamma \\
= & {\left[\Delta_{m}\right]_{3}^{3} \cdot \Gamma-\gamma_{\bar{V}} \int \mathrm{d}^{4} x \rho_{a \mu} C_{a}^{\mu} } \\
& +\chi \partial_{\xi} \int \mathrm{d}^{4} x\left(\gamma_{A} \rho_{a \mu} A_{a}^{\mu}+\gamma_{\bar{V}} \rho_{a \mu} V_{a}^{\mu}-\gamma_{G} \sigma_{a} c_{a}+\gamma_{F}(\bar{Y} \Psi-\bar{\Psi} Y)\right)
\end{aligned}
$$

By means of the BRS-varying gauge parameter we find that the BRS-symmetric operators which are not BRS variations have to appear with $\xi$-independent coefficient functions (cf. (2.44)),

$$
\partial_{\xi} \beta_{g}=0,
$$

whereas all the other coefficient functions are not restricted concerning their gauge parameter dependence. This result holds to all orders of perturbation theory.

Furthermore, testing (6.3) on the gauge fixing condition (4.21) one is able to determine $\gamma_{\bar{V}}$ :

$$
\gamma_{\bar{V}}=\bar{z}\left(\beta_{g}-\gamma_{A}\right)+\left(2 \gamma_{A} \xi \partial_{\xi}-\beta_{g} g \partial_{g}\right) \bar{z}
$$

In the conventional background field approach (cf. (5.20)) the parameter $\bar{z}$ takes the definite value $\bar{z}=1$ to all orders. In this case the expression for $\gamma_{\bar{V}}$ simplifies to

$$
\gamma_{\bar{V}}=\beta_{g}-\gamma_{A}
$$


However, in order to have a $\xi$-independent normalization condition for the background vector 2-point functions (cf. (5.13)), we keep $\bar{z}$ as an additional free parameter of the model in the following.

The RG equation is derived in a completely analogous manner. We just state the final result:

$$
\begin{aligned}
& \left(\kappa \partial_{\kappa}+\tilde{\beta}_{g}\left(g \partial_{g}-\int \mathrm{d}^{4} x\left(V_{a \mu} \frac{\delta}{\delta V_{a \mu}}+C_{a \mu} \frac{\delta}{\delta C_{a \mu}}\right)\right)\right. \\
& -\tilde{\gamma}_{A}\left(\int \mathrm{d}^{4} x\left(A_{a \mu} \frac{\delta}{\delta A_{a \mu}}-\rho_{a \mu} \frac{\delta}{\delta \rho_{a \mu}}-B_{a} \frac{\delta}{\delta B_{a}}-\bar{c}_{a} \frac{\delta}{\delta \bar{c}_{a}}\right)+2 \xi \partial_{\xi}+2 \chi \partial_{\chi}\right) \\
& -\tilde{\gamma}_{\bar{V}} \int \mathrm{d}^{4} x V_{a \mu} \frac{\delta}{\delta A_{a \mu}}-\tilde{\gamma}_{G} \int \mathrm{d}^{4} x\left(c_{a} \frac{\delta}{\delta c_{a}}-\sigma_{a} \frac{\delta}{\delta \sigma_{a}}\right) \\
& -\tilde{\gamma}_{F} \int \mathrm{d}^{4} x\left(\bar{\Psi} \frac{\vec{\delta}}{\delta \bar{\Psi}}+\frac{\overleftarrow{\delta}}{\delta \Psi} \Psi \bar{Y} \frac{\vec{\delta}}{\delta \bar{Y}}-\frac{\overleftarrow{\delta}}{\delta Y} Y\right) \\
& \left.+\chi \partial_{\xi} \tilde{\gamma}_{A}\left(\int \mathrm{d}^{4} x\left(\bar{c}_{a} \frac{\delta}{\delta B_{a}}\right)-2 \xi \partial_{\chi}\right)\right) \Gamma \\
& =\tilde{\gamma}_{\bar{V}} \int \mathrm{d}^{4} x \rho_{a \mu} C_{a}^{\mu} \\
& +\chi \partial_{\xi} \int \mathrm{d}^{4} x\left(\tilde{\gamma}_{A} \rho_{a \mu} A_{a}^{\mu}+\tilde{\gamma}_{\bar{V}} \rho_{a \mu} V_{a}^{\mu}-\tilde{\gamma}_{G} \sigma_{a} c_{a}+\tilde{\gamma}_{F}(\bar{Y} \Psi-\bar{\Psi} Y)\right)
\end{aligned}
$$

Please note that due to the physical normalization conditions chosen no $\beta$-function in connection with a physical mass appears in (6.7). Again, by means of a BRS-transforming gauge parameter, one proves the gauge parameter independence of $\tilde{\beta}_{g}$,

$$
\partial_{\xi} \tilde{\beta}_{g}=0
$$

to all orders of the perturbative expansion. The test of (6.7) on the gauge condition determines $\tilde{\gamma}_{\bar{V}}$ in analogy to $(6.5)$ to be:

$$
\tilde{\gamma}_{\bar{V}}=\bar{z}\left(\tilde{\beta}_{g}-\tilde{\gamma}_{A}\right)+\left(2 \tilde{\gamma}_{A} \xi \partial_{\xi}-\tilde{\beta}_{g} g \partial_{g}\right) \bar{z}
$$

Finally, we turn to the equation describing the $\xi_{0}$-dependence of the theory. Expanding $\xi_{0} \partial_{\xi_{0}} \Gamma$ like before in the basis of BRS-symmetric operators, some of these operators are prohibited to appear right from the beginning because the normalization conditions fixing the corresponding coefficient functions do not depend on $\xi_{0}$. Having already performed these trivial tests on some of the normalization conditions we find:

$$
\begin{aligned}
& \left(\xi_{0} \partial_{\xi_{0}}+\beta_{g}^{\xi_{0}}\left(g \partial_{g}-\int \mathrm{d}^{4} x\left(V_{a \mu} \frac{\delta}{\delta V_{a \mu}}+C_{a \mu} \frac{\delta}{\delta C_{a \mu}}\right)\right)-\gamma_{\bar{V}}^{\xi_{0}} \int \mathrm{d}^{4} x V_{a \mu} \frac{\delta}{\delta A_{a \mu}}\right) \Gamma \\
= & -\gamma_{\bar{V}}^{\xi_{0}} \int \mathrm{d}^{4} x \rho_{a \mu} C_{a}^{\mu}+\chi \partial_{\xi} \gamma_{\bar{V}}^{\xi_{0}} \int \mathrm{d}^{4} x \rho_{a \mu} V_{a}^{\mu}
\end{aligned}
$$


Testing this equation on (5.13), also $\gamma_{\bar{V}}^{\xi_{0}}$ has to vanish:

$$
\gamma_{\bar{V}}^{\xi_{0}}=\bar{z} \beta_{g}^{\xi_{0}}-\beta_{g}^{\xi_{0}} g \partial_{g} \bar{z}-\xi_{0} \partial_{\xi_{0}} \bar{z}=0
$$

The first equality in the above equation results from the test of $(\sqrt{6.10})$ on the gauge condition (4.21). Therefore the $\beta$-function of the $\xi_{o}$-equation is determined by the parameter $\bar{z}:$

$$
\beta_{g}^{\xi_{0}}=\left(\bar{z}-g \partial_{g} \bar{z}\right)^{-1} \xi_{0} \partial_{\xi_{0}} \bar{z}
$$

and

$$
\beta_{g}^{\xi_{0}}=\xi_{0} \partial_{\xi_{0}} \delta \bar{z}^{(1)}+O\left(\hbar^{2}\right)=-\frac{N g^{2}}{4 \cdot 32 \pi^{2}} \xi_{o} \partial_{\xi_{o}}\left(\left(\xi_{o}-1\right)\left(\xi_{o}+7\right)\right)+O\left(\hbar^{2}\right)
$$

Here we have used the explicit result of the 1-loop order (5.19).

In the background field gauge and in 1-loop order the $\beta$-function of the $\xi_{o}$-equation is independent of the explicit form of the 1-loop Ward identity and is given in the normalization (5.13) as well as in the conventional normalization (5.21) by:

$$
\beta_{g}^{\xi_{0}}=-\frac{N g^{2}}{4 \cdot 32 \pi^{2}} 2 \xi_{o}\left(\xi_{o}+3\right)+O\left(\hbar^{2}\right)
$$

Hence using (6.11) the $\xi_{0}$-equation reads

$$
\left(\xi_{0} \partial_{\xi_{0}}+\beta_{g}^{\xi_{0}}\left(g \partial_{g}-\int \mathrm{d}^{4} x\left(V_{a \mu} \frac{\delta}{\delta V_{a \mu}}+C_{a \mu} \frac{\delta}{\delta C_{a \mu}}\right)\right)\right) \Gamma=0
$$

and, again, the $\beta$-function $\beta_{g}^{\xi_{0}}$ is $\xi$-independent to all orders,

$$
\partial_{\xi} \beta_{g}^{\xi_{0}}=0
$$

but depends - as seen from the explicit expression (6.13) - on $\xi_{o}$.

Determining the Green functions in the MS or $\overline{\mathrm{MS}}$ scheme a gauge normalization point is not explicitly introduced, but, of course, one has also implicitly chosen a gauge parameter value $\xi_{o}$ where the background field 2-point function is normalized. The $\xi_{o}$-equation cannot be derived in these schemes, since $\xi_{o}$-dependence is hidden, but nevertheless, if we would do so, then its $\beta$-function coincides with the one, we have calculated in (6.14), at a special value of $\xi_{o}$. 


\section{Construction of a RG equation invariant charge}

In the following we will restrict ourselves for reasons of brevity to the massless theory in which case the RG equation and the CS equation coincide. The generalization to the massive theory is easy and straightforward.

According to the observations (for QED) in section 2 the object $Q_{g}\left(p^{2}, \kappa^{2}, \xi_{o}\right)$ we are looking for has (at least) to satisfy the homogeneous RG equation,

$$
\left(\kappa \partial_{\kappa}+\tilde{\beta}_{g} g \partial_{g}\right) Q_{g}\left(p^{2}, \kappa^{2}, \xi_{o}\right)=0
$$

has to be gauge parameter independent,

$$
\partial_{\xi} Q_{g}\left(p^{2}, \kappa^{2}, \xi_{o}\right)=0
$$

and also well-normalized:

$$
\left.Q_{g}\left(p^{2}, \kappa^{2}, \xi_{o}\right)\right|_{p^{2}=\kappa^{2}}=g^{2}
$$

In the nonabelian theory considered in this paper, the invariant charge $Q_{g}$ in addition has to obey the homogeneous $\xi_{0}$-equation. This last requirement will be discussed in detail in the following section.

In a first step we will now show that an object satisfying (7.1)-(7.3), i.e. a RG equation invariant charge, can be uniquely defined in a perturbative way out of a certain combination of vector and background vector self-energies. This will be done by explicitly constructing the lowest orders of the self-energies $\tilde{\Pi}\left(p^{2}, \kappa^{2}, \xi_{o}\right)$. From this expression the invariant charge is constructed as in QED,

$$
Q_{g}\left(p^{2}, \kappa^{2}, \xi_{o}\right)=\frac{g^{2}}{1+\tilde{\Pi}\left(p^{2}, \kappa^{2}, \xi_{o}\right)}, \quad \tilde{\Pi}=O(\hbar)
$$

and $\tilde{\Pi}$ has to satisfy the following equations (see also (2.14), (7.1)-(7.3)):

$$
\begin{aligned}
\left(\kappa \partial_{\kappa}+\tilde{\beta}_{g} g \partial_{g}-2 \tilde{\beta}_{g}\right)\left(1+\tilde{\Pi}\left(p^{2}, \kappa^{2}, \xi_{o}\right)\right) & =0 \\
\partial_{\xi} \tilde{\Pi}\left(p^{2}, \kappa^{2}, \xi_{o}\right) & =0 \\
\left.\left(1+\tilde{\Pi}\left(p^{2}, \kappa^{2}, \xi_{o}\right)\right)\right|_{p^{2}=\kappa^{2}} & =1
\end{aligned}
$$

Before starting with the explicit construction of $\tilde{\Pi}$ we want to collect some formulae useful for the subsequent calculations and discussions. Differentiation of the RG equation twice with respect to $A_{a \mu}$, evaluation of the resulting expression for all fields as well as $\chi$ set equal to zero and projection onto the transversal part yields:

$$
\kappa \partial_{\kappa} \Gamma_{A A}^{T}+\tilde{\beta}_{g} g \partial_{g} \Gamma_{A A}^{T}-2 \tilde{\gamma}_{A} \Gamma_{A A}^{T}-2 \tilde{\gamma}_{A} \xi \partial_{\xi} \Gamma_{A A}^{T}=0
$$


( $\Gamma_{A A}^{T}$ is defined in (2.38).) Similarly one obtains

$$
\begin{gathered}
\kappa \partial_{\kappa} \Gamma_{A V}^{T}+\tilde{\beta}_{g} g \partial_{g} \Gamma_{A V}^{T}-\tilde{\beta}_{g} \Gamma_{A V}^{T}-\tilde{\gamma}_{A} \Gamma_{A V}^{T}-2 \tilde{\gamma}_{A} \xi \partial_{\xi} \Gamma_{A V}^{T}-\tilde{\gamma}_{\bar{V}} \Gamma_{A A}^{T}=0 \\
\kappa \partial_{\kappa} \Gamma_{V V}^{T}+\tilde{\beta}_{g} g \partial_{g} \Gamma_{V V}^{T}-2 \tilde{\beta}_{g} \Gamma_{V V}^{T}-2 \tilde{\gamma}_{A} \xi \partial_{\xi} \Gamma_{V V}^{T}-2 \tilde{\gamma}_{\bar{V}} \Gamma_{A V}^{T}=0
\end{gathered}
$$

by testing the RG equation with respect to $A_{a \mu}, V_{b \nu}$ or twice with respect to $V_{a \mu}$, respectively.

Using (7.8)-(7.10) an easy calculation shows that the quantity $\bar{\Gamma}$, defined by

$$
\bar{\Gamma}=\bar{z}^{2} \Gamma_{A A}^{T}+2 \bar{z} \Gamma_{A V}^{T}+\Gamma_{V V}^{T}
$$

obeys to all orders of perturbation theory the following homogeneous RG equation:

$$
\kappa \partial_{\kappa} \bar{\Gamma}+\tilde{\beta}_{g} g \partial_{g} \bar{\Gamma}-2 \tilde{\beta}_{g} \bar{\Gamma}-2 \tilde{\gamma}_{A} \xi \partial_{\xi} \bar{\Gamma}=0
$$

This expression is valid independently from the choice of the parameter $\bar{z}$. From (5.18), (5.19) and (5.22), respectively, we read off that $\bar{\Gamma}$ is gauge parameter dependent in 1-loop order and, hence, is not an appropriate object for defining a RG-invariant charge.

We would like to mention already now that, on the other hand, $\bar{\Gamma}$, as defined in (7.11), does indeed satisfy the correct $\xi_{0}$-equation of an invariant charge for arbitrary $\bar{z}$ (see section 8):

$$
\xi_{0} \partial_{\xi_{0}} \bar{\Gamma}+\beta_{g}^{\xi_{0}} g \partial_{g} \bar{\Gamma}-2 \beta_{g}^{\xi_{0}} \bar{\Gamma}=0
$$

We now turn to the explicit determination of a RG invariant charge by constructing order to order in perturbation theory $\tilde{\Pi}\left(p^{2}, \kappa^{2}, \xi_{o}\right)$. In 1-loop order the RG equation for $\tilde{\Pi}(7.5)$ reads:

$$
\kappa \partial_{\kappa} \tilde{\Pi}^{(1)}-2 \tilde{\beta}_{g}^{(1)}=0
$$

A closer look to (7.8)-(7.10), these equations also written in strict 1-loop order, shows that up to local counterterms such an object is uniquely determined by:

$$
p^{2} \tilde{\Pi}^{(1)}=\Gamma_{A A}^{T(1)}+2 \Gamma_{A V}^{T(1)}+\Gamma_{V V}^{T(1)}
$$

This quantity is gauge parameter independent, only if we state the normalization condition (5.13) of the generalized background gauge (cf. (5.18)): The $\kappa$-dependent part of (or the nonlocal contributions to) $\tilde{\Pi}^{(1)}$ is $\xi$-independent since the $\beta$-function is $\xi$-independent (6.8). Furthermore, the $\kappa$-independent part of (or the local contributions to) $\tilde{\Pi}^{(1)}$ is also gauge parameter independent due to the normalization condition (5.13). Hence, $1+\tilde{\Pi}^{(1)}$ can be used to construct a RG-invariant charge up to 1-loop order according to eq. (7.4). We want to conclude the treatment of the 1-loop approximation by establishing the relation between $\tilde{\Pi}^{(1)}$ and $\bar{\Gamma}^{(1)}$. A simple calculation shows that:

$$
\begin{gathered}
p^{2} \tilde{\Pi}^{(1)}=\bar{\Gamma}^{(1)}-2 p^{2} \delta \bar{z}^{(1)} \\
\left(\text { with } \bar{z}=1+\delta \bar{z}^{(1)}+\ldots\right)
\end{gathered}
$$


This means that the unwanted but unavoidable gauge parameter dependence of $\bar{\Gamma}^{(1)}$ can be (and, in fact, is) removed by means of the local contribution $-2 p^{2} \delta \bar{z}^{(1)}$, i.e. by means of the additional free parameter $\bar{z}$. The full power of having at hand this additional freedom when looking for a RG equation invariant charge, however, only turns out in higher orders.

The construction of these higher orders of $\tilde{\Pi}$ is done in a recursive manner and follows exactly the strategy which was outlined above for the 1-loop order. Here we skip the details of the calculation and just present the final result for 2- and 3-loop order:

$$
\begin{aligned}
p^{2} \tilde{\Pi}^{(2)}= & \Gamma_{A A}^{T(2)}+2 \Gamma_{A V}^{T(2)}+\Gamma_{V V}^{T(2)} \\
& -p^{2} \tilde{\Pi}^{(1)}\left(1-g \partial_{g}\right) \delta \bar{z}^{(1)}+\Gamma_{A A}^{T(1)}\left(1-2 \xi \partial_{\xi}\right) \delta \bar{z}^{(1)} \\
p^{2} \tilde{\Pi}^{(3)}= & \Gamma_{A A}^{T(3)}+2 \Gamma_{A V}^{T(3)}+\Gamma_{V V}^{T(3)} \\
& -p^{2} \tilde{\Pi}^{(1)}\left(1-g \partial_{g}\right) \delta \bar{z}^{(2)}+\Gamma_{A A}^{T(1)}\left(1-2 \xi \partial_{\xi}\right) \delta \bar{z}^{(2)} \\
& -p^{2} \tilde{\Pi}^{(2)}\left(1-g \partial_{g}\right) \delta \bar{z}^{(1)}+\Gamma_{A A}^{T(2)}\left(1-2 \xi \partial_{\xi}\right) \delta \bar{z}^{(1)} \\
& -\frac{1}{2} \frac{1}{p^{2}}\left(p^{2} \tilde{\Pi}^{(1)}-\Gamma_{A A}^{T(1)}\right)\left(p^{2} \tilde{\Pi}^{(1)}\left(1-g \partial_{g}\right) \delta \bar{z}^{(1)}-\Gamma_{A A}^{T(1)}\left(1-2 \xi \partial_{\xi}\right) \delta \bar{z}^{(1)}\right) \\
& -\frac{1}{2} \Gamma_{A A}^{T(1)} \tilde{\Pi}^{(1)}\left(1+2 \xi \partial_{\xi}\right)\left(1-g \partial_{g}\right) \delta \bar{z}^{(1)} \\
+ & \frac{1}{4} p^{2} \tilde{\Pi}^{(1)^{2}}\left(1+g \partial_{g}\right)\left(1-g \partial_{g}\right) \delta \bar{z}^{(1)}+\frac{1}{4} \frac{1}{p^{2}} \Gamma_{A A}^{T(1)^{2}}\left(1+2 \xi \partial_{\xi}\right)\left(1-2 \xi \partial_{\xi}\right) \delta \bar{z}^{(1)}
\end{aligned}
$$

The invariant charge constructed from $\tilde{\Pi}=1+\sum_{n=1}^{3} \tilde{\Pi}^{(n)}$ is well normalized according to the normalization conditions (5.12) and (5.13). Gauge parameter independence is proven order by order by using gauge parameter independence of $\beta$-functions and the normalization conditions. Hence, we have succeeded in defining a RG-invariant charge for QCD (i.e. an object satisfying (7.1)-(7.3)) up to 3-loop order as a unique combination of 2-point functions.

\section{Compatibility with the $\xi_{0}$-equation?}

One of the main results of the abstract construction is the observation, that a proper scheme-independent definition of QCD-parameters asks for the introduction of a normalization gauge parameter $\xi_{o}$. For nonabelian gauge theories in linear gauges the $\xi_{o}$ and its corresponding differential equation have been introduced in [2] (see also section 2, (2.39)). In the present paper we have shown, that also in the background field gauge it is not possible to avoid the introduction of $\xi_{o}$ by means of the Ward identity (see sections 4, 5 and 
(5.13), (5.21), respectively).5 The dependence of Green functions on $\xi_{o}$ is governed by a partial differential equation, which ensures $\xi_{o}$-independence of physical quantities, in a similar manner as the $\mathrm{RG}$ equation ensures normalization parameter independence of physical quantities. In the background field gauge the $\xi_{o}$-equation was derived in section 6 , see 6.15$)$.

It is obvious that the invariant charge should not only be a normalization point independent object but also a $\xi_{o}$-independent object, and therefore should satisfy both equations, the $\mathrm{RG}$ equation and the $\xi_{o}$-equation, i.e.:

$$
\left(\xi_{0} \partial_{\xi_{0}}+\beta_{g}^{\xi_{0}} g \partial_{g}\right) Q_{g}\left(p^{2}, \kappa^{2}, \xi_{o}\right)=0
$$

In terms of $1+\tilde{\Pi}$, the "inverse" of $Q_{g}$, 8.1) reads:

$$
\left(\xi_{0} \partial_{\xi_{0}}+\beta_{g}^{\xi_{0}} g \partial_{g}-2 \beta_{g}^{\xi_{0}}\right)\left(1+\tilde{\Pi}\left(p^{2}, \kappa^{2}, \xi_{o}\right)\right)=0
$$

In the preceding section we have uniquely constructed a gauge parameter independent RGinvariant charge in terms of $\tilde{\Pi}$. An easy calculation, however, shows that this invariant charge fails to fulfill (8.2). In fact, one finds

$$
\xi_{0} \partial_{\xi_{0}} \tilde{\Pi}^{(1)}=0 \quad \text { and } \quad \xi_{0} \partial_{\xi_{0}} \tilde{\Pi}^{(2)}=0
$$

i.e. the lowest orders of the RG-invariant charge does not explicitly depend on $\xi_{o}$. Since, however, the $\beta$-function $\beta_{g}^{\xi_{0}}$ is non-vanishing outside the Landau gauge, see (6.13), the invariant charge depends on $\xi_{0}$ via the coupling.

From the construction it is obvious that it is not possible to construct a RG-invariant charge, which has well-defined normalization conditions and fulfills at the same time the differential $\xi_{o}$-equation, out of a combination of 2-point functions in linear background gauges. Only the Landau gauge seems to be distinguished from other gauges since in this gauge the RG- $\beta$-function of the gauge parameter $\tilde{\beta}_{\xi}=\xi \tilde{\gamma}_{A}$ and the gauge coupling $\beta$-function $\beta_{g}^{\xi_{o}}$ of the $\xi_{o}$-equation vanish.

We want to mention that we did indeed succeed in a conventional way to define a RGinvariant charge in 1-loop order, which is related to the Pinch technique self-energies and is gauge parameter independent. Furthermore, we were also able to continue this invariant charge to higher orders (explicitly up to 3-loop). Thereby we have used arguments similar to those given in the construction of Pinch technique self-energies in higher orders [17. However, by a careful analysis of invariant counterterms, normalization conditions and the $\chi$-enlarged Slavnov-Taylor identity we found, that QCD has to be complemented

\footnotetext{
${ }^{3}$ We want to mention, that in the Abelian Higgs model the $\chi$-enlarged ST identity could be fulfilled without introducing $\xi_{o}$ by using the local Ward identity and on-shell conditions, see [14, 16].
} 
by a gauge parameter normalization and a respective partial differential equation. It is this equation which makes the construction of an invariant charge impossible in the conventional linear background gauge. The $\xi_{o}$-equation has been not considered up to now in explicit calculations since in such calculations one frequently sticks to special invariant schemes like the MS and $\overline{\mathrm{MS}}$ scheme. Therefore we claim that a first step towards a deeper and better understanding of the construction of an invariant charge requires a careful abstract solution of the $\mathrm{RG}$ and the $\xi_{o}$-equation.

\section{Conclusions}

In the present paper we studied gauge parameter dependence in Yang-Mills theories including background fields. These were not introduced ad hoc but rather as a tool to couple systematically to currents which otherwise would have prohibited the existence of a local Ward identity. As a concrete application we tried to construct a gauge invariant charge made up from 2-point functions of the quantum and the background vector fields, thus combining suggestions from the pinch technique with the background field method. Up to and including three loops we showed that such an object exists and is characterized as:

- being solution of the homogeneous renormalization group equation (7.1),

- being gauge parameter independent (7.2),

- coinciding with the coupling at a normalization point (7.3).

In our abstract renormalization scheme independent approach it is, however, apparent that a complete set of normalization conditions which is compatible with the gauge parameter dependence (controlled algebraically to all orders) requires a normalization gauge parameter $\xi_{0}$ (see sections 4,5 ) which is on the same footing as the momentum scale normalization parameter $\kappa$. It gives rise to an analogous parametric differential equation (6.15). Since the otherwise perfect candidate for an effective charge fails to satisfy the corresponding homogeneous equation (8.1) we consider the issue of constructing an effective charge in Yang-Mills theories as not yet finally settled. 


\section{References}

[1] H. Kluberg-Stern and J.B. Zuber, Phys. Rev. D12 (1975) 467, 482.

[2] O. Piguet and K. Sibold, Nucl. Phys. B253 (1985) 517.

[3] B.S. DeWitt, Phys. Rev. D162 (1967) 1195.

G. 't Hooft, Acta Universitatis Wratislavensis 368 (1976) 345.

H. Kluberg-Stern and J.B. Zuber, Phys. Rev. D12 (1975) 482 and 3159.

[4] L.F. Abbott, Nucl. Phys. B185 (1981) 189.

[5] A. Denner, G. Weiglein, and S. Dittmaier, Nucl. Phys. B440 (1995) 95 and Phys. Lett. B333 (1994) 420.

[6] N.J. Watson, Nucl. Phys. B494 (1997) 388.

J. Papavassiliou, E. de Raffael, and N.J. Watson, Nucl. Phys. B503 (1997) 79.

[7] J.M. Cornwall, Phys. Rev. D26 (1982) 1453.

J.M. Cornwall and J. Papavassiliou, Phys. Rev. D40 (1989) 3474.

J. Papavassiliou, Phys. Rev. D41 (1990) 3179.

J. Papavassiliou, Ringberg Electroweak 1995:0247-262, hep-ph/9504384, and references therein.

[8] G. Degrassi and A. Sirlin, Phys. Rev. D46 (1992) 3140.

[9] V.I. Zakharov, Nucl. Phys. B385 (1992) 452.

M. Beneke and V.I. Zakharov, Phys. Rev. Lett. 69 (1992) 2472.

[10] W. Celmaster and R. J. Gonsalves, Phys. Rev. D20 (1979) 1420.

[11] A.I. Davydychev, P. Osland, O.V. Tarasov, Phys. Rev. D54 (1996) 4087.

[12] S. Hashimoto, J. Kodaira, Y. Yasui, K. Sasaki, Phys. Rev. D50 (1994) 420.

[13] P. A. Grassi, Nucl. Phys. B462 (1996) 524.

[14] R. Häußling and E. Kraus, Z. Phys. C75 (1997) 739.

[15] R. Kallosh, Nucl. Phys. B78 (1974) 293.

[16] R. Häußling and S. Kappel, to appear in Eur. J. Phys. C, hep-th/9707165.

[17] J. Papavassiliou and A. Pilaftsis, Phys. Rev. D54 (1996) 5315. 